\title{
Research on Personalized Information Recommendation of Library
}

\author{
Bo $\mathrm{He}^{1, \text { a }}$, Hongyuan Zhang ${ }^{1, \mathrm{~b}}$ \\ ${ }^{1}$ School of Computer Science and Engineering, ChongQing University of Technology, \\ ChongQing, China \\ aheboswnu@sina.com, ${ }^{\mathrm{b}} 464234870 @ q q . c o m$
}

Keywords: Library, Personalized information recommendation, User profiles

\begin{abstract}
Personalized information recommendation service of library had becoming an important research task. This paper divided library users into new users and other users. It proposed the recommendation strategy based on user segmentation. On the base of these, it designed a personalized information recommendation system of library, namely, PIRSL. The experimental results indicate that the recommendation strategy is feasible.
\end{abstract}

\section{Introduction}

The research of personalized information service of library has made a progress during these years, the most important of which is personalized information recommendation[1]. The traditional recommendation strategy could not satisfy current library and users' needs. This paper establishes user profiles and puts forward a recommendation strategy based on user segmentation[2], designs a personalized information recommendation system of library, namely, PIRSL.

\section{User profiles}

Establishment of user profiles[3] is the base of realizing personalized information recommendation of library.

Definition 1 User profiles can be described as an aggregation, namely, $U_{i}=\left\{\left(d_{i, 1}, w_{i, 1}\right)\right.$, $\left.\left(d_{i, 2}, w_{i, 2}\right), \ldots,\left(d_{i, n}, w_{i, n}\right)\right\}$, and $d_{i, j} \in T . T=\left\{t_{1}, t_{2}, \ldots, t_{m}\right\}$ is an aggregation of user interest subjects, $w_{i, j} \in[0,1]$ is the weight of user interest subjects, and $w_{i, 1}+w_{i, 2}+\ldots+w_{i, n}=1$.

User profiles are constructed by three ways:

(1)According to the information of registration, PIRSL gets elementary user profiles.

(2)PIRSL picks up user profiles according to mining request of user.

(3)PIRSL adjusts user profiles according to the estimation of mining results.

When a user registers, PIRSL makes the user answer a series of questions. The system produces a user interest tree according to the answers. The interest subjects is more and more specific from the root to the leaf of the interest tree. The process from root to leaf is the process of discovering user interest subjects. The more deep the layer of user interest tree is, the bigger of the weight of the user interest subjects is. According to human-computer interaction, PIRSL takes out primary user interest subjects and establishes user profiles.

When a user inputs mining request each time, the system picks up user interest subjects from it and renews user profiles. The algorithm is described as follows.

Alogrithm 1 Renewal algorithm of the user profiles

Input: mining request of user is $I$, constant $c$, and $0 \leq c \leq 1 / n$, the previous user profiles $\left\{\left(d_{i, 1}, w_{i, 1}\right)\right.$, $\left.\left(d_{i, 2}, w_{i, 2}\right), \ldots\left(d_{i, n}, w_{i, n}\right)\right\}$.

Output: the renewed user profiles $\left\{\left(d_{i, 1}, w_{i, 1}\right),\left(d_{i, 2}, w_{i, 2}\right), \ldots\left(d_{i, n}, w_{i, n}\right)\right\}$.

Methods: According to the following steps.

(1) Picking up effective vocabulary $L$ from $I$.

(2) If $L \in\left\{d_{i, 1}, d_{i, 2}, \ldots d_{i, n}\right\}$, suppose $L=d_{i, p}$, then transfer to (3), or else (4).

(3) $\left(d_{i, p}, w_{i, p}+c\right) \longrightarrow\left(d_{i, p}, w_{i, p}\right)$, then transfer to (6). 
(4) Add $L$ into $\left\{d_{i, 1}, d_{i, 2}, \ldots d_{i, n}\right\}$, suppose $d_{i, q}=L$, then $w_{i, q}=\min \left\{w_{i, j} \mid 1 \leq j \leq n\right\}$. If $c>w_{i, q}$, then transfer to(5), or else (6).

(5) $(L, c) \longrightarrow\left(d_{i, q}, w_{i, q}\right)$.

(6) If there is other effective vocabulary form $I$, then transfer to (1).

(7) $w_{i, j}=w_{i, j} / \sum_{k=1}^{n} w_{i, k}$.

\section{The recommendation strategy based on user segmentation}

The user of library is described as follow.

User $=\langle$ NewUser, OtherUser $\rangle$

NewUser $=\{$ User $\mid$ User_current-User_start $\leqslant \sigma\}$

OtherUser $=\{$ User $\mid$ User_current-User_start $>\sigma\}$

User_start is the first time of visit.

User_current is the current time.

$\sigma$ is threshold.

The recommendation strategy based on user segmentation provides two kinds of recommendation algorithms. One is the intelligent information recommendation algorithm based on user model clustering, namely IRUMC algorithm[4]. The other is Web intelligent information recommendation method based on collaborative filtering, namely WIIRM algorithm[5]. IRUMC algorithm fits new users. WIIRM algorithm fits other users.

The recommendation strategy based on user segmentation is described as follows.

if (the user belongs to new users)

PIRSL adopts IRUMC algorithm;

else

PIRSL adopts WIIRM algorithm;

\section{Personalized information recommendation system of library}

On the base of user profiles and the recommendation strategy based on user segmentation, this paper designs a personalized information recommendation system of library, namely, PIRSL. PIRSL includes the interface module, the preprocessing module, the mining module and the on-line recommendation module and. PIRSL is described as fig 1. 


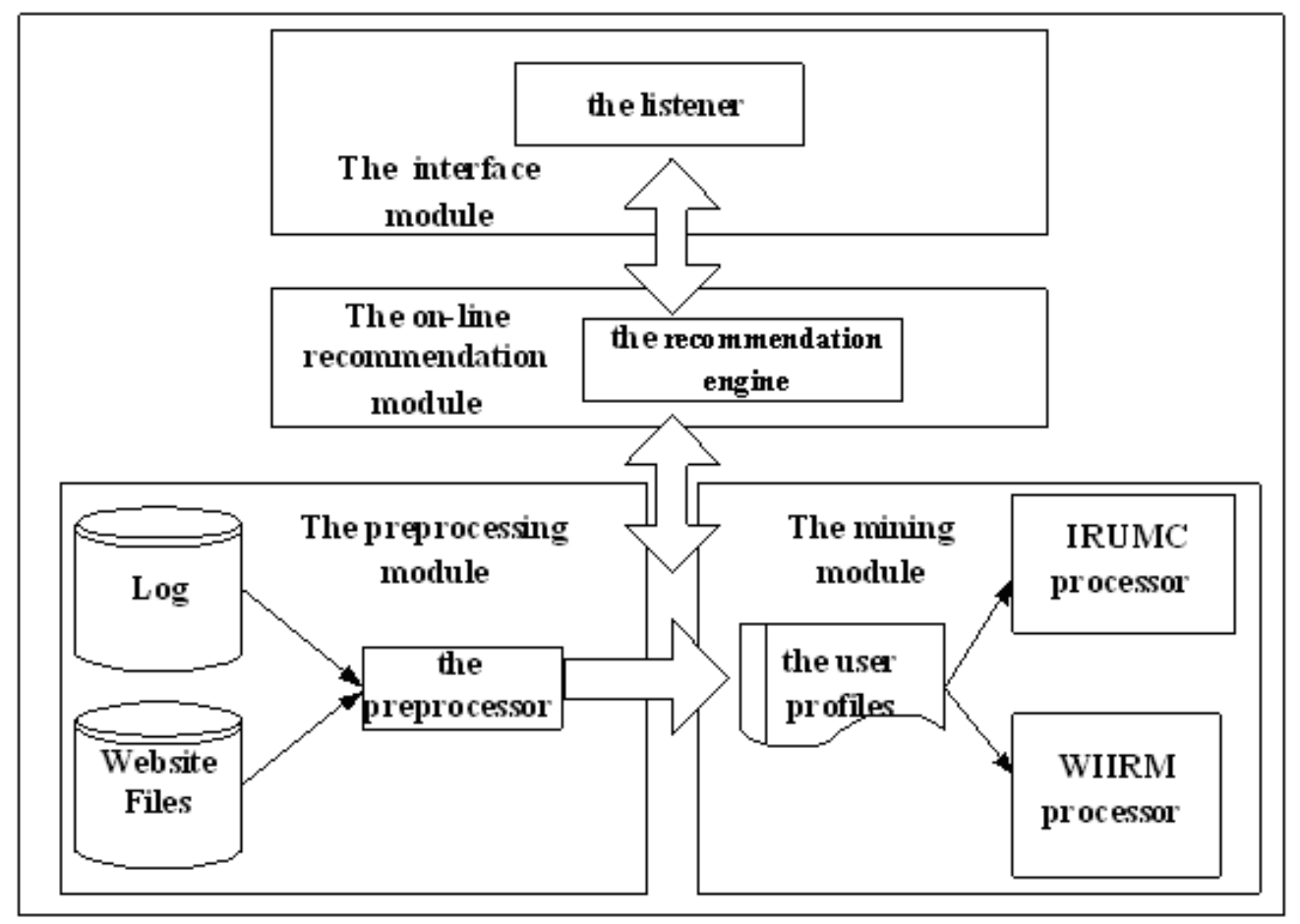

Fig.1 FIRSL

The interface module includes the listener. The interface module is the interface of user and system. The preprocessing module includes the preprocessor. The preprocessing mainly includes data cleaning, user recognition, session identification, path supplementation and user pattern recognition, etc.

The mining module includes IRUMC processor and WIIRM processor. IRUMC processor treats data by IRUMC algorithm. WIIRM processor treats data by WIIRM algorithm.

The on-line recommendation module includes the recommendation engine. The recommendation engine can gain recommendation sets by recommendation algorithms.

\section{Related Experiments}

The experimental data comes from the log of a website in July 2010. PIRSL adopts the IRUMC algorithm and WIIRM algorithm, as fig 2.

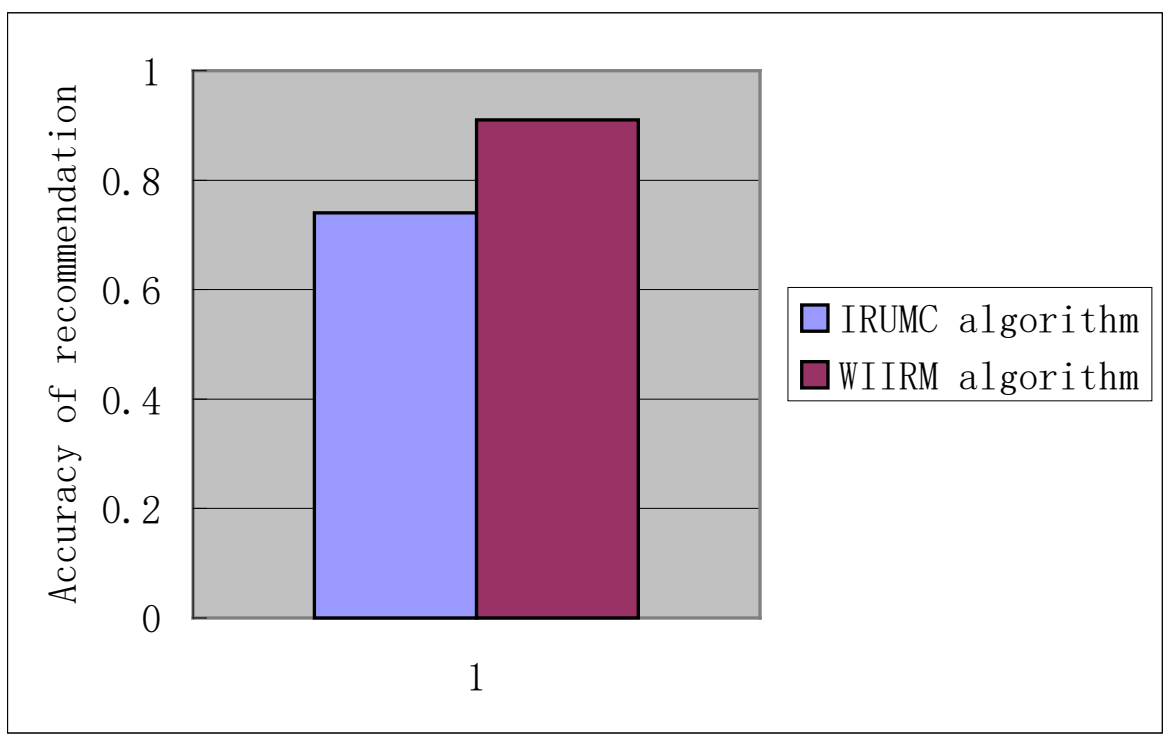

Fig.2 Comparison of two recommendation algorithms

The accuracy of two recommendation algorithm is 0.74 and 0.91 . Hence, IRUMC fits new users. WIIRM algorithm fits other users. The experimental results indicate that the recommendation 
strategy based on user segmentation is feasible.

\section{Conclusion}

The paper designs a personalized information recommendation system of library, namely, PIRSL. The experimental results indicate that the recommendation strategy is feasible. It is believed that personalized information recommendation of library will have a magnificent future.

\section{Acknowledgment}

This research is supported by the social science planning and cultivation project of chongqing under grant No.2014PY50 and the humanities and social science research project of chongqing municipal education commission under grant No. 15SKG131. This research is supported by the fundamental and advanced research projects of chongqing under grant No. CSTC2013JCYJA40039 and the national statistical science research project under grant No. 2015LZ22.

\section{References}

[1] Marko B. An Adapative Web Page Recommendation Service[C]. 1th International Conference on Autonomous Agents, Marina del Rey, Feburuary 1997.

[2] Xu M, Qiu YH, Qiu J, and Wang XF. Segmenting your users in recommender system[C]. Proceeding of the IASTED International Conference: Modeling and Simulation'2003.Palm Springs, CA,USA. ACTA Press, pages 584-588.

[3] Zeng C, Xing CX, Zhou LZ. A survey of personalization[J]. Jounal of Software, 2002,13(10):1952 1961(in Chinese with English abstract).

[4] He B, Yang W, Zhang JX, Wang Y. Intelligent information recommendation algorithmbased on user model clustering[J]. Computer engineering and design. 2006,27(13):2360-2361. (in Chinese with English abstract).

[5] He B. Web Intelligent Information Recommendation Method Based on Collaborative Filtering[J].library and information service, 2010,54(19):115-118. (in Chinese with English abstract).

[6] He B, Wang Y, Yang W and Chen Y. Fast Algorithm for Mining Global Frequent Itemsets Based on Distributed Database [C]. Rough Sets and Knowledge Technology, Chongqing, 2006, 415-420.

[7] He B. Fast Mining of Global Maximum Frequent Itemsets in Distributed Database [J]. Control and Decision, 2011,26(8):1214 1218. (in Chinese with English abstract) 